\title{
Nanotechnology, Nanoparticles and Nanoscience: A New Approach in Chemistry and Life Sciences
}

\author{
Dan Tshiswaka Dan \\ Zhejiang Normal University, Jinhua, China \\ Email: dantshis07@gmail.com
}

How to cite this paper: Dan, D.T. (2020) Nanotechnology, Nanoparticles and $\mathrm{Na}$ noscience: A New Approach in Chemistry and Life Sciences. Soft Nanoscience Letters, 10, 17-26.

https://doi.org/10.4236/snl.2020.102002

Received: March 1, 2020

Accepted: April 27, 2020

Published: April 30, 2020

Copyright (อ 2020 by author(s) and Scientific Research Publishing Inc. This work is licensed under the Creative Commons Attribution International License (CC BY 4.0).

http://creativecommons.org/licenses/by/4.0/

\begin{abstract}
Nanotechnologies, nanoparticles and nanomaterials, which are part of everyday life today, are the subject of intense research activities and a certain amount of media coverage. In this article, the concepts of nanotechnologies, nanoparticles and nanosciences are defined and the interest in this scale of the matter is explained by specifying in particular the particular properties of nanoobjects. Large-scale applications of nanoparticles, particularly in the field of chemicals, everyday life and catalysis are presented.
\end{abstract}

\section{Keywords}

Nanoscience and Nanoparticles

\section{Introduction}

Nano sciences and nanotechnologies have been the subject of numerous works for more than twenty years, within and at the interface of multiple scientific disciplines, such as physics, chemistry, biology, engineering sciences or human sciences and social. Nanotechnology research is raising high hopes because of the particular properties of matter at the nanoscale that allow new functions unimagined to be envisaged [1]. Manufacturing, observing and manipulating nano-objects, studying and understanding their properties and their interactions with their environment, in particular with living organisms, modeling and simulating them, integrating them into communicating systems, these have been and still are the major challenges scientists essential to meet in order to develop numerous and considerable applications, but in a controlled manner. The applications of nanotechnology are increasingly important in the life of every individual, for industry and commerce, for health and society. Today, research and development work are exploding on the applications of nanotechnologies in the 
fields of energy, chemistry and sensors, materials, information and communications, biology and of medicine, of the environment. This rich landscape should not obscure other aspects, including, as a counterpoint to the advantages, the new risks of nanotechnologies for health, the environment, respect for private life, or even further, the evolutions of the species and human. The challenges to be met are therefore immense and the competition between large countries is appearing fiercer and fiercer.

\section{Two approaches to nano}

From the 1930s to the 1980s, the emergence of powerful imaging methods made it possible to probe and even manipulate matter on a scale unattainable with a traditional light microscope. This has aroused a very strong enthusiasm from various scientific communities. The fabrication and study of small objects with a few tens to a few thousand atoms then became a major challenge. However, from the beginning of the 20th century, the tools of chemistry (organic synthesis and polymerization) and biology (peptide synthesis) routinely manufactured objects of a few nanometers, generally carbon-based, by assembling smaller bricks or molecules (so-called ascending or "bottom-up" route). So, for a chemist, a nanoparticle, which contains a few thousand atoms, is a big object! At the origin of Nanotechnology are scientists who have taken the problem backwards. They have developed new tools to miniaturize traditional macroscopic matter: electron, tunneling and atomic force microscopes, nano-lithography [2], etc. This is the so-called descending or "top-down" path: making a single nanometric object such as a small cluster of metallic atoms, then imaging and manipulating it using the technological tool, constitutes the heart of this new science [3]. The combination of direct imagery that is widely understood by everyone, with the remote-controlled manipulation, atom by atom, of artificial structures, has made the notion of nanoparticle concrete and has nourished the imagination of a whole generation: nano-robots and nano-machines operating in our daily environment, even inside the human body, are the key dreams of this time. In practice, the miniaturization of printed circuits, in other words the exponential increase in the capacities of memories and processors, constitutes the great technological success of these techniques.

However, making everyday materials often requires more rustic methods. Even if atomic manipulation has progressed immensely over the past 30 years, culminating in the making of the smallest film in the world ("A Boy and His Atom" [4]), this century will probably not reach mass production of nano-robots functional with these tools, for many reasons. First, atomic manipulation only gives access to one object at a time and requires a lot of time and effort. Then, the objects are manufactured in conditions far from the ambient conditions (low temperature and low pressure) to avoid any interference with the environment and are often not stable outside their place of manufacture. It is therefore more of a proof of concept-very elegant-than a technological solution.

The two paths to the nano world, top-down and bottom-up, are sometimes 
taken together to lead to original structures. For example, to manufacture nano-cars and other molecular machines, organic chemistry is used to prepare, from small molecules, the building blocks (wheels, axle, etc.). Then the pieces are assembled into "supramolecular" objects (the pioneers of this approach are none other than J.M-Lehn and J.P. Sauvage [5], both Nobel Prize winners in Chemistry). These assemblies are then studied using top-down imaging tools, for example atomic force microscopy. This can give rise to unusual sporting events, such as nano-car races, to the delight of all [6]!

\section{Nanotechnology in Daily Life}

The nanoparticles and nanomaterials found today on the market and in industry are produced by large-scale methods, directly related to the manufacturing processes of macroscopic materials: materials with high mechanical quality, cosmetics, smart glasses, etc. lithium battery electrodes, etc. In terms of tonnage, these mainly concern the following products (examples of application fields are given in brackets): silica nanoparticles (food additive, tire reinforcement), titanium dioxide (cosmetics), alumina (food additive, adjuvant in the medical field), zinc oxide (cosmetic) and cerium (paint), carbon nanotubes (mechanical reinforcement for sporting goods), fullerenes and carbon black (inks, battery electrodes in lithium), silver nanoparticles (anti-bacterial, low energy loss glasses) and iron (soil decontamination), dendrimer [7] (therapeutic) and nano-clays (absorbents). For all of these products, the raw material used is common whether it is a mineral material (e.g. silica) or an organic molecule. It is the nanoscale shaping that is the key to the new application. For example, the fiber structures of carbon nanotubes give rise to new mechanical properties, which do not exist in graphite [8] (Figure 1). Likewise, the use of titanium dioxide as an absorber of ultraviolet radiation in sunscreens requires submicron particles to preserve the texture and appearance of the cream.

Thus, the nanoscopic nature does not make it possible to identify, on its own, the potential of nanotechnologies nor the new risks associated with nanomaterials currently on the market. We must remember both the idea of small size and that of the presence of a new property as a consequence of this reduced size. Moreover, this is how most operational and institutional definitions proceed.
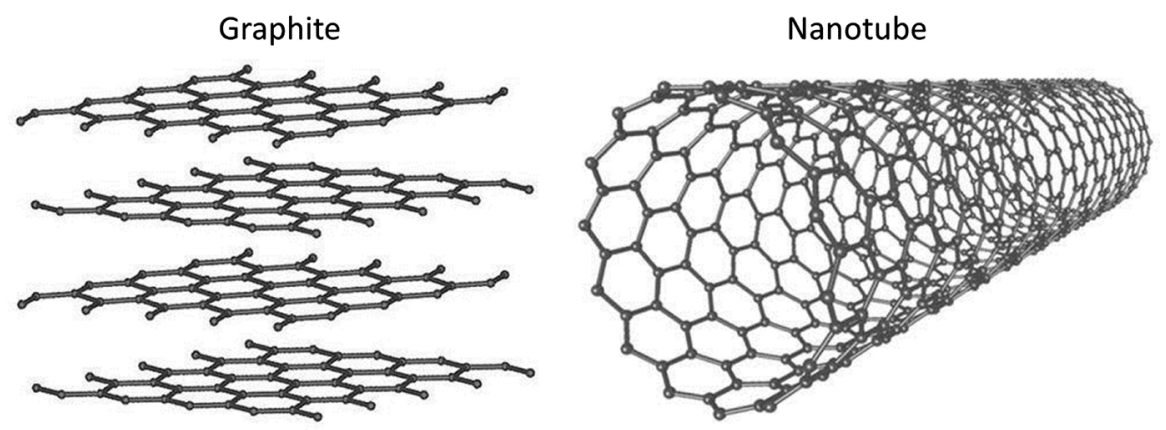

Figure 1. Carbon in two forms: graphite (sheet) and nanotube (source: illustration adapted from Wikipedia France). 
Nanotechnologies have an impact in many areas of everyday life: food, energy, environment, health, safety, transport, etc. Without creating a pervert-style catalog, we will cite its main applications:

- Automotive: reinforced and lighter materials; exterior paints with color effects, brighter, anti-corrosion, anti-scratch, and anti-dirt; fuel additives improving their combustion; more durable and recyclable tires

- Aeronautics and space: sensors for optimizing engine performance; ice detectors on airplane wings etc.

- Electronics and telecommunications: high density memories; miniaturized processors; light sensors, handheld computers; super-fast computers and programs; wireless technologies; flat screens etc.

- Chemistry and materials: pigments; ceramic powders; corrosion inhibitors; fillers in polymers; catalysts; antibacterial or ultra-resistant textiles and coatings etc.

- Construction: self-cleaning and anti-pollution cements, self-cleaning and anti-dirt windows; paintings; varnish; glues; sealants etc.

- Food industry: coloring agents, anti-caking agents, emulsifiers; active packaging extending the shelf life etc.

- Health: drugs and active agents; anti-allergenic medical adhesive surfaces; tailor-made drugs targeted to specific organs (or cells); bio-compatible materials for implants; oral vaccines; medical imaging...

- Cosmetics: transparent sun creams; abrasive toothpaste etc.

- Leisure: ski wax, rackets and tennis balls etc.

- Energy: new generation photovoltaic cells; high storage capacity batteries; storage of fuel hydrogen; smart windows; more efficient insulating materials etc.

- Defense: detectors of chemical and biological agents; miniaturized surveillance systems; ultra-precise guidance systems; light and/or self-healing textiles

- Environment and ecology: reduction of carbon monoxide emissions by catalysis; desalination of seawater; specific chemical analyzers; etc.

In total, nanotechnologies address a global market that exceeded 1000 billion dollars in 2015.

And they now have their place in modern manufacturing production, led by a growing number of industrialized countries, including Germany, Australia, Canada, China, the United States, France, Japan and the Republic of Korea

\section{Usage in Chemistry}

\subsection{Surface Chemistry on Nanoparticles}

To keep the case of gold, a striking example is the ability of gold nanoparticles to cause chemical reactions between molecules in a gas mixture to which they are exposed. Solid gold is an extremely inert metal; that is why it is used for precision work, for standards or to coat other metals more susceptible to oxidation or 
corrosion. On the contrary, gold nanoparticles are very reactive. They "catalyze" the reaction of oxidation of carbon monoxide to carbon dioxide at low temperature $\left(-70^{\circ} \mathrm{C}\right)$, reaction of the equation $\mathrm{CO}+1 / 2 \mathrm{O}_{2}$ to $\mathrm{CO}_{2}$. This means that they facilitate and accelerate this reaction which would require otherwise a significant energy input (heating, etc.) to take place.

This remarkable result dating from the 1980s is at the origin of a new golden age (it is the case to say it) for catalysis [9]. It is a lesser-known example of the role of nanoparticles in the development of clean and energy-efficient technologies. Likewise, platinum nanoparticles are incorporated into car catalytic converters to clean up gaseous emissions from combustion engines by accelerating the destruction of the most harmful chemical compounds (nitrogen and carbon monoxides).

\subsection{Usage in Catalysis}

\section{Catalysis: a field at the heart of nano sciences}

In fact, the general public often ignores the role that nanoparticles play in the field of catalysis, probably because this field itself-however essential in terms of economic and environmental impact-is not well understood. Catalytic phenomena are indeed ubiquitous. The enzyme system of living organisms is composed of molecular catalysts at work at all times to carry out chemical reactions at room temperature, which would be extremely slow without a catalyst, at room temperature. For example, the photosynthesis of green plants, that is to say the transformation of carbon dioxide into plant matter, requires breaking bonds between carbon atoms and oxygen atoms and forming new ones between carbon atoms to change from gaseous carbon dioxide to organic molecules. The simplified equation for the transformation of $\mathrm{CO}_{2}$ into glucose by photosynthesis is written: $6 \mathrm{CO}_{2}+6 \mathrm{H}_{2} \mathrm{O}$ to $\mathrm{C}_{6} \mathrm{H}_{12} \mathrm{O}_{6}+6 \mathrm{O}_{2}$.

Similarly, the respiration of mammals, that is to say the consumption of oxygen to produce energy, involves sequences of catalytic reactions perfectly adjusted in time and space thanks to millennia of biological evolution. For its part, the industry has used since its foundation artificial catalysts-inspired by organic systems or not-to manufacture products for agriculture (nitrogen fertilizers), consumer goods (plastics, polymers) or transport (fuels), or for recycling and depollution.

It is estimated that $90 \%$ of the products manufactured in the world have undergone at least one catalytic step during their manufacture. To make the best use of the energy and raw materials that the planet offers us, manufacturers and scientists are seeking to improve existing catalysts, to make them more efficient, less expensive and less toxic. Nanoparticles and nanomaterials are catalysts of choice because they offer a wide range of compositions and properties, which can be adjusted at will to the desired reaction. The chemical reactions take place on the surface of the nano catalysts: their large state of division guarantees maximum use of the material. In addition, the size of nanoparticles influences 
their way of catalyzing chemical reactions. This makes it possible to control reactions more finely, and in particular, to promote the formation of certain products over others as required.

For more than a century, many industrial processes have used nanoparticles. Among the most essential, two of them are described below: they are still at the heart of very active research to adapt to the ever-increasing constraints on energy consumption and raw materials.

\subsection{Nitrogen to Feed the Planet}

Another example showing the weight and the potential of catalysis in energy and environmental questions is the Haber-Bosch process, dating from 1909. Here, it is a question of using dinitrogen, the most abundant gas in the atmosphere, and convert it to ammonia $\left(\mathrm{NH}_{3}\right)$ which is the source to make nitrogen fertilizers. The reaction involved has the equation $\mathrm{N}_{2}+3 \mathrm{H}_{2}$ to $2 \mathrm{NH}_{3}$; it therefore requires breaking the nitrogen-nitrogen triple bond of $\mathrm{N}_{2}$, a very difficult step, and then reacting the nitrogen atoms with hydrogen atoms (supplied by hydrogen gas). This process is widely used worldwide because it guarantees crop yields through the use of synthetic fertilizers. Modern catalysts are nanoparticles of iron or ruthenium, deposited on a suitable support (silica, alumina, etc.) and improved by additives such as potassium: these nanomaterials here also have a very specific structure, optimized at all stages of the design.

Despite everything, the operating temperatures and pressures are still high for this process (around $400^{\circ} \mathrm{C}$ and 200 bar). By way of comparison, the nitrogenase enzymes of green plants perform the same transformation at $25^{\circ} \mathrm{C}$ and under atmospheric pressure, but with a much lower yield unfortunately... The volume of nitrogenous fertilizers required each year on the whole planet is such that this industrial process alone consumes around 1\% of the world's energy produced! It is trivial to say that optimizing its performance, even by a few tenths of a percent, would help reduce the energy footprint of our civilization. This is why research and development, from the most fundamental to the most applied, is still extremely active in nano catalysis.

\section{Nanoparticles: Curious Objects}

The scientific curiosity aroused by nanomaterials stems as much from their new intrinsic properties as from their range of applications. In the case of semiconductors, we are witnessing the appearance of a quantum effect on the nanometric scale: while a macroscopic light-emitting diode has a fixed color, nanoparticles of the same material will have a color depending directly on their size. The same is true for other fundamental properties, such as the melting point: a gold nanoparticle $3 \mathrm{~nm}$ in diameter becomes liquid at twice the temperature of solid gold.

\section{Nanometric Gold Coloration}

New physical phenomena are also appearing at the nanometric scale. Also, in 
the case of gold, a ruby red coloration appears for particles of around ten nanometers in diameter. This effect is due to the particular behavior of the electronic cloud which surrounds the nanoparticle. This resonates with certain light frequencies and preferentially absorbs them. Glass craftsmanship used gold in the red coloring of glass and stained glass, called Cassius purple, long before the manufacturing process was understood to generate nanoparticles (Figure 2). A famous object, the Cup of Lycurgus, features this deep red color to illustrate a scene from Greek mythology in which King Lycurgus attacks Dionysus, god of the vine and excess.

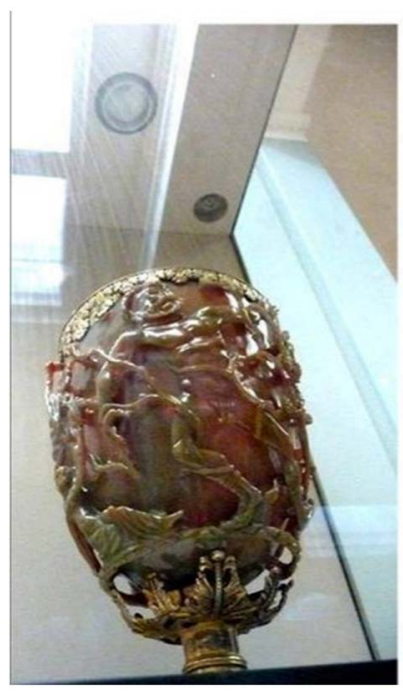

Usual lighting

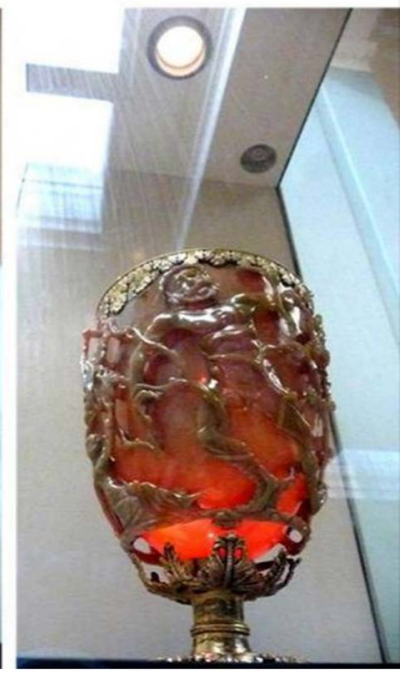

transmission lighting

Figure 2. The Lycurgus cup, on display at the British Museum, contains gold and silver nanoparticles. If it is lit from its sides (usual lighting), it appears green. If it is lit from the inside, in other words in "transmission" (the object is then between the lamp and the eye), it appears red. [Photo credit and copyright: S. Carenco].

The scientific curiosity aroused by nanomaterials stems as much from their new intrinsic properties as from their range of applications. In the case of semiconductors, we are witnessing the appearance of a quantum effect on the nanometric scale: while a macroscopic light-emitting diode has a fixed color, nanoparticles of the same material will have a color depending directly on their size. The same is true for other fundamental properties, such as the melting point: a gold nanoparticle $3 \mathrm{~nm}$ in diameter becomes liquid at twice the temperature of solid gold.

\section{Nanotechnology Dangers}

While the applications of these Lilliputian objects and technologies are booming and have invaded our daily lives, their uses present risks:

- Sanitary facilities for consumers and for professionals involved in their production, in particular due to the strong penetrating capacity of nanoparticles in cell tissues due to their size, which allows them to overcome certain natu- 
ral barriers. While all nanomaterials are not dangerous, uncertainties remain as to their possible effects (inflammatory, respiratory, cardiovascular or neurological) which are insufficiently documented;

- Environmental nanoparticles can also disperse and persist in the environment and we do not know the impact. Subject to confirmation, laboratory studies indicate that microorganisms, invertebrates, and plants may be affected by exposure to certain nanomaterials.

The Scientific Committee on Emerging and New Health Risks (SCISSEN), which works within the European Commission, has highlighted the inadequacy of existing methods for the assessment of risks to health and the environment.

There are currently no specific regulations governing the handling of nanomaterials in France and Europe. It is the principles of health protection which are applicable, as well as the texts devoted to the marketing of chemical substances, drugs, cosmetic products or food.

Since January 2013, manufacturers, importers and distributors of nanoproducts in France only have the obligation to declare the identity, quantities and uses of nanomaterials to the National Food Safety Agency, environment and work (ANSES).

At European level, the regulations governing nanomaterials, in cosmetics, in biocidal products or in food are generally considered insufficient by associations for the protection of consumers, health, the environment and workers.

As far as consumer information is concerned, "nano" labeling is compulsory in Europe for three categories of products.

- Cosmetics, since July 2013: The Cosmetics Regulation requires manufacturers to mention the presence of nanomaterials in the list of cosmetic ingredients: the labeling rule provides that the term "nano" is indicated in square brackets after the name of the ingredient. In the case of $\mathrm{TiO}_{2}$ for example: Titanium dioxide [nano].

- Biocides, since September 2013: The Biocides Regulation also requires that the label indicate the presence of nanomaterials in biocidal products, with the term "nano" in parentheses, after the name of the ingredient and "the possible specific risks that are related "must be mentioned.

- Food products, since December 2014 (theoretically): the INCO Regulation had first provided for the obligation to affix the label "nano" in square brackets, before the name of the ingredient concerned. But pressure from industrialists in the sector to reduce this obligation has delayed the entry into force of this measure. The obstacle was lifted at the end of October 2015, with the vote of the Novel Foods Regulation.

\section{Advantages and Disadvantages}

\subsection{Advantages}

Many potential applications and advantages include:

- Advances in disease treatments, such as cancer 
- Better imaging and diagnostic equipment

- Energy-efficient products such as fuel and solar cells

- Improvements in manufacturing that allow for durable, light-weight, efficient production tools

- Improved electronic devices, including transistors, LED and plasma displays and quantum computers

- Nanorobots can be used to rebuild the ozone layer, clean polluted areas and lesson dependence on non-renewable energy sources

\subsection{Disadvantages Include}

- The potential dangers to humans and the environment

- Loss of manufacturing and agricultural jobs

- Economic market crashes related to a potential lower value of oil due to more efficient energy sources and gold or diamonds, materials that can be reproduced with molecular manipulation

- Accessibility of weapons of mass destruction

- Improved atomic weaponry

- The cost of research and products made from nanoparticles

\section{Nano Science and Nanoparticles: A Real Scientific Challenge}

On a very small scale, some physical properties appear, others disappear. Some are improved or disturbed: lightness, resistance, conductivity, thermal, adhesion, magnetism or even aesthetics etc. Impossible then to rely on the classical laws of physics to observe, understand and manufacture nano objects. The nanometer domain is, in fact, governed by the rules of quantum mechanics.

The miniaturization of chip components is a major scientific issue for the processing of data from this research. It promises vastly amplified power for all electronics, from computing speeds to the storage capacities of hard drives. An Intel computer processor, which contained 2300 transistors in 1971, now contains 50,000 times as many. And soon, the development of a quantum computer could emerge thanks to the nanocrystals, nanowires, nanocomposites and molecular electronics under study.

For this, researchers must overcome the physical limit of integrated circuits on silicon (reached in 2010 according to forecasts of Moore's law) by creating new optical architectures, and not electrical.

\section{Conclusions}

Nanomaterials don't stop there; they are going beyond what we think.

These examples from the field of daily life, chemistry and catalysis illustrate the essential role played by nanomaterials, even before the appearance of the word nano sciences. Many other fields today rely on these complex and original compounds to progress: photovoltaics, therapeutic vectorization, energy storage 
and lithium batteries, etc. Their horizons go beyond the sometimes-minimalist framework of the current debate surrounding "nanos". Due to the diversity of their nature and their applications, these new materials raise many ethical and toxicological questions of prime importance, which we have not extensively discussed here. Their potential goes beyond applications such as sunscreens (titanium oxide nanoparticles) or antibacterial socks (silver nanoparticles), which are much more often mentioned in the "mainstream" press. The submerged part of the iceberg (catalysis, clean energies, but also the successful understanding of the properties of matter etc.) seems more relevant than ever to us in a global reflection on "nanos".

\section{Conflicts of Interest}

The author declares no conflicts of interest regarding the publication of this paper.

\section{References}

[1] Internet: Royalsociety.Org (Last Checked 23 June 2019). https://royalsociety.org/ /media/Royal Society Content/policy/publications/2004/ 9693.pdf

[2] Joachim.C. and Plévert, L. (2008) Nanosciences: La révolution invisible. Seuil, France. https://doi.org/10.14375/NP.9782020867030

[3] Internet: A Boy and His Atom: The World's Smallest Movie (Last Checked 19 July 2020).

http://www.research.ibm.com/articles/madewithatoms.shtml; http://www.youtube.com/watch?v=oSCX78-8-q0\&feature=player embedded

[4] Internet: Resources Published on Culture Sciences Chemistry: Nobel Prize in Chemistry 2016: Molecular Machines and from Chemical Topology to Molecular Machines-Conference by Jean-Pierre Sauvage (Last Check 27 June 2020).

https://theconversation.com/conversation-avec-jean-pierre-sauvage-nobel-de-chimi e-et-machines-moleculaires-124789

[5] Internet: Nanocar, the First-Ever Race of Molecule-Cars (Last Checked 25 Jully 2020). http://nanocar-race.cnrs.fr

[6] Internet: Surendra Nimesh, in Gene Therapy: Potenial Application of Nanotechnologiy, 2013. (Last Checked 08 June 2020 )

https://www.sciencedirect.com/topics/pharmacology-toxicology-and-pharmaceutic al-science/dendrimers

[7] Haruta, M., Kobayashi, T., Sano, H. and Yamada, N. (1987) Novel Gold Catalysts for the Oxidation of Carbon Monoxide at a Temperature far below $0^{\circ} \mathrm{C}$. Chemistry Letters, 16, 405-408. https://doi.org/10.1246/cl.1987.405

[8] Thompson, D. (2007) Michael Faraday's Recognition of Ruby Gold: The Birth of Modern Nanotechnology. Gold Bulletin, 40, 267-269.

http://link.springer.com/article/10.1007\%2FBF03215598

[9] Michael, F. (1857) Experimental Relations of Gold (and Other Metals) to Light. Philosophical Transactions of the Royal Society of London, 147, 145-181. https://doi.org/10.1098/rstl.1857.0011 